\title{
TIERRAS ESTATALES Y DESIGUALDAD DE GÉNERO EN URUGUAY
}

\author{
State lands and gender inequality in Uruguay
}

\author{
Paula Florit O`Neill \\ paufloron@gmail.com \\ Universidad de la República - Uruguay
}

\author{
Maximiliano Piedracueva \\ maxipc85@gmail.com \\ Universidad de la República - Uruguay
}

Recibido: 01-03-2017

Aceptado: 30-04-2017

\section{Resumen $^{1}$}

En Uruguay, el Instituto Nacional de Colonización (INC) es la institución encargada de la adjudicación de tierra por parte del Estado, orientado a la distribución racional de la tierra y su acceso por parte de la población rural más vulnerable. A partir del año 2005, atravesó una reforma que jerarquizó su rol en las políticas de Estado y gestó un nuevo modelo de adjudicación de tierras: las experiencias asociativas. Ese mismo año, el nuevo gobierno impulsó la transversalización de género en el Estado, impulso que anclara en el INC a partir del 2013. Este artículo analiza desde una perspectiva de género la reforma refundacional y la nueva política de tierras, develando la ceguera de género de la reforma y evidenciando desafíos pendientes para la transversalización de género en el diseño de la principal modalidad de adjudicación de tierras públicas.

Palabras clave: Género, tierra, políticas públicas, transversalización.

\begin{abstract}
In Uruguay, the National Institute of Colonization is the institution resposible for the adjudication of land by the State, aimed at the rational distribution of land and its access by the most vulnerable rural population. Since 2005, the INC went through a reform that prioritized its role in the policies of the State and created a new model of land adjudication: associative experiences of colonization. In that same year, the new government promoted gender mainstreaming in the State, impulse that took roots in the INC from 2013 onwards. This article analyzes from a gender perspective the refounding reform and the new land policy, unveiling the gender blindness of the reform and highlighting pending challenges for gender mainstreaming in the design of the main public land adjudication modality.
\end{abstract}

Palabras clave: Gender, land, public policies, mainstreaming.

\footnotetext{
${ }^{1}$ Este documento fue parcialmente presentado a las jornadas de Investigación y Extensión de FHUCE en el año 2013 y constituye una reproducción parcial y actualizada de la monografía de egreso del Diploma en Género y Políticas Públicas de FCS presentada en el año 2014.
} 


\section{Introducción}

El presente artículo se centra en el proceso de incorporación de la perspectiva de género en la reforma atravesada por el Instituto Nacional de Colonización de Uruguay, y su subproducto la política de adjudicación de tierras asociativa. La investigación se desarrolló desde la perspectiva del gender mainstreaming aplicando esta perspectiva de política pública a la colonización, en el entendido de que la tierra constituye un recurso fundamental en el desarrollo de los países. El acercamiento abona a un análisis desde la mirada del acceso y control como categorías reveladoras en torno a la tierra, desde la perspectiva de género.

El artículo se estructura en siete secciones, la actual introducción donde se da cuenta del encuadre y estructura del documento, así como los materiales y métodos de la investigación, y seis apartados subsiguientes. En la sección dos se profundiza en la perspectiva de gender mainstreaming desde la cual se trabaja. En la sección tres se introduce la política de colonización pre existente a la reforma del instituto y se encuadra el problema de la desigualdad de género en el acceso a la tierra en Uruguay. La cuarta sección enuncia el proceso de reforma del INC ubicando el carácter ciego de la etapa fundacional y la inflexión hacia una perspectiva de género a partir del año 2013. El quinto apartado caracteriza en términos de diseño la principal política actual de colonización, las experiencias asociativas, producto de la reforma iniciada en 2005 . La sexta sección analiza esta política a través de las categorías de acceso y control de la tierra, a partir de la información emergida de los datos de la encuesta a experiencias asociativas de la Regional Salto de INC. Finalmente la séptima sección enuncia las reflexiones resultantes de los apartados anteriores en torno a los desafíos actuales para una transversalización de género de la política de tierras públicas.

A efectos de realizar el presente trabajo se combinaron diversas técnicas de carácter cualitativo y cuantitativo, procesándose información pre existente y generándose fuentes primarias. El análisis de todas ellas permitió configurar una imagen sobre la política actual de colonización y los cambios introducidos por la reforma en el INC a partir de 2005. Fueron parte de este trabajo las siguientes prácticas y técnicas: (i) Análisis documental de las leyes 11.029, 18.187 y 18.756 referidas a los formatos colonizadores y los cometidos del INC, el Plan Estratégico 2010 - 2014 de INC, y la revisión de las actas de directorio de los años 2007 a 2013; (ii) Entrevistas a informantes calificadas de INC; (iii) Procesamiento secundario de información del Censo General Agropecuario de MGAP (2000) y del Censo de Colonos y colonias de INC (2005 - 2006); (iv) Producción de una encuesta a todas las colonias asociativas comprendidas en la regional Salto de INC. Esta quinta estrategia se desarrolló habida cuenta de que no existía información primaria sobre el acceso y uso de tierras por parte de las mujeres en el marco de las experiencias asociativas como nuevo modelo colonizador, acotándose la encuesta a una regional de INC, la regional Salto, por ser una de las regionales con mayor número de experiencias 
asociativas. La encuesta abarcó a 322 personas pertenecientes a 126 familias colonas y se realizó en el trimestre comprendido entre diciembre de 2013 y febrero de 2014.

\section{Gender mainstreaming}

El presente documento se construye bajo un supuesto no necesariamente $\operatorname{acordado}^{2}$ ni por la academia ni por el movimiento feminista, a saber, la asunción de que es posible y necesario transformar al Estado de forma que abandone la reproducción de patrones sexistas para actuar como un detractor de la desigualdad de género a la interna del aparato estatal y hacia la sociedad.

La propuesta del gender mainstreaming parte del supuesto de que no existen políticas neutrales, sino que todas están generizadas e inciden diferencialmente en mujeres y varones, habitualmente reforzando parámetros de un sistema asimétrico y centrado en el varón. Esta perspectiva no es privativa de las políticas vinculadas a las mujeres, a la sexualidad y a las familias, sino que la cuestión de género es transversal a todas las políticas porque es constitutiva de toda una sociedad que hace del sexo un argumento central para la atribución de roles y acciones en todas las dimensiones de la vida cotidiana. Esta interpretación supone que las estrategias que desarrollan políticas marginales vinculadas específicamente con las mujeres nunca podrán ser eficaces en la reversión de las condiciones de desigualdad, porque ésta se gesta en la corriente principal de las políticas.

En consecuencia, la propuesta de las transversalización supone transformar el Estado instalando una nueva cultura institucional según la cual todas las políticas públicas, en todas sus fases, deben ser analizadas e influidas por una perspectiva de género orientada a revertir el orden imperante en la sociedad y en el propio aparato estatal. Para ello, esta mirada debe abandonar un lugar marginal, orientado a las mujeres y exclusivo de funcionarios/as especializados/as, para abarcar las políticas públicas centrales del Estado y ser incorporada al funcionariado en general, revirtiendo las condiciones y estatus de la cuestión de género e incidiendo tanto en la vida de las mujeres y como en la de los varones (Incháustegui y Ugalde, 2004).

En la concreción del gender mainstreaming un aspecto a tener en cuenta es el contexto histórico específico que determina la oportunidad o no de realizar adelantos en la cuestión de género. Guzmán y Montaño (2012) destacan especialmente que las políticas públicas son el resultado de luchas de poder, vinculadas a la interpretación disímil de la realidad y de las formas como debe intervenirse en la misma. En consecuencia, el proceso de institucionalización de las políticas de género supone la pugna por la ubicación de esta cuestión en la agenda del Estado mediante una lucha por la interpretación del rol de éste, de la realidad vinculada a varones y mujeres, y de las posibilidades político institucionales concretas en tiempos históricos determinados.

\footnotetext{
${ }^{2}$ Véase Pateman, 1996.
} 
Un segundo aspecto se relaciona con la constitución del triángulo de terciopelo. Según Walby (2004) en el camino del gender mainstreaming existe una polémica sobre quiénes deben desarrollar la estrategia de transversalidad de género, en particular si el desarrollo de éstas políticas debe estar a cargo de especialistas o si este debe ser desarrollado con importante participación de la ciudadanía y especialmente de los movimientos feministas. Sobre este punto, Rigat-PFlaum (2008) señala las limitaciones y alcances de un proceso de análisis técnico de una estructura estatal necesariamente burocrática y política. En esta perspectiva, el propio Estado es impulsor de la revisión y objeto de la misma, siendo sus estructuras y los procesos de su funcionariado el objeto de análisis y revisión. Consecuentemente, señala la autora se generan tensiones en el análisis al entrar en contienda las aspiraciones y orientaciones técnicas del gender mainstreaming, las resistencias de la estructura estatal y el funcionariado y la viabilidad política. En este sentido, Rigat-Pflaum recupera como una orientación valiosa -validada por la experiencia-, la concepción de que la viabilidad de la instalación de un proceso de gender mainstreaming depende de la existencia de un acuerdo entre el movimiento feminista, las académicas feministas y quienes hacen las políticas (decisores, técnicos/as, operadores). Red de voluntades e impulsos que fuera denominada por Woodward "el triángulo de terciopelo" 3 (Woodward, 2003).

\section{La colonización previa y la desigualdad de género en el acceso a la tierra}

Las políticas de distribución de tierras en el ámbito nacional encuentran su antecedente directo en el Reglamento Provisorio de la Provincia Oriental para el Fomento de su Campaña y Seguridad de sus Hacendados del año 1815. Desde entonces y a través de distintos mecanismos, el Estado uruguayo ha contemplado la importancia de esta distribución principalmente con el objetivo de poblar el medio rural y contener el acaparamiento de tierras. En el año 1945 se realiza el Congreso Nacional de la Colonización en el cual participan diversos actores públicos y privados, y es a partir de dicho congreso que surgen las primeras ideas de generar una institución que tuviera como objetivo único la colonización. De ese modo en el año 1948, y a través de la ley 11.029 se crea el Instituto Nacional de Colonización (INC), estableciéndose la generación de una institucionalidad pública orientada a velar por la distribución de la tierra.

En términos de sus funciones, desde sus comienzos el INC operó principalmente como canalizador entre demandas de tierras y su oferta desde el Estado; en este sentido el INC otorgaba parcelas a colonos y sus familias en distintas modalidades (individual, cooperativa, colectiva; en propiedad, en arrendamiento, en aparcería). Históricamente la modalidad principal de otorgamiento de tierras era de modo individual y a través de cesión de derechos de propiedad o contratos de

\footnotetext{
3 "Velvet triangle" en el original.
} 
arrendamiento. Hasta el año 2005 la asignación se realizaba principalmente por el otorgamiento de parcelas individuales a grupos familiares a los cuales se accedía a través de un persona aspirante denominado en toda la documentación del INC "el colono"- que aparecía como titular único de un predio que arrendaba -con o sin derecho a compra- para el usufructo de su familia.

\begin{abstract}
“Artículo 59.- Los aspirantes a colonos deben reunir las siguientes condiciones: A) Tener 18 años cumplidos, para lo cual y a los efectos de esta ley se les declara en mayoría de edad; B) Poseer conocimientos y aptitudes suficientes para el género de explotación a que vayan a dedicarse; C) Poseer condiciones personales y hábitos de vida que el Instituto considere satisfactorios.

Artículo 60.- Se dará preferencia, dentro de las condiciones señaladas en el artículo anterior, a los aspirantes a colonos: A) Que posean mejores aptitudes y condiciones personales; B) Que se organicen en Cooperativas o Sindicatos; C) Que hayan acreditado mejores aptitudes en los núcleos de capacitación; D) Que sean agricultores desalojados o estén pendientes de desalojo, o que se encuentren en las condiciones previstas en el artículo 20; E) Que posean familia, de preferencia si ella es apta para colaborar en el trabajo del predio; F) Que sean hijos de colonos;

Artículo 61.- Serán obligaciones principales de los colonos: A) Hacer efectivos los pagos dentro de los plazos fijados en dinero o en especie según se hubiera convenido; B) Trabajar directamente el predio y habilitarlo con su familia, salvo, en este último caso, que la colonia esté organizada o se organice bajo el sistema de viviendas agrupadas en poblados" (Ley 11.029. Reproducción parcial de los artículos).
\end{abstract}

La tarea del INC estaba orientada, en consecuencia, a un uso social de la tierra, encuadrada en el supuesto de que la familia productora estaba radicada en la parcela de colonización. La relación tierra - colono - familia atraviesa entonces la historia de la colonización en Uruguay, dándose por supuesto en la ley la coincidencia de la unidad productiva y la reproductiva o doméstica. Asimismo, el implícito en la ley 11.029 y en su accionar a través de asignación a un integrante "el colono", es que las familias son unidades homogéneas donde el poder y las decisiones se distribuyen en un colectivo, no siendo relevante o necesaria la titulación de los recursos a nombre de todas las personas que integrantes. Subyace, en consecuencia, la noción patriarcal del jefe de familia y su orientación a la protección de todo el núcleo.

No obstante, la relevancia de la propiedad al interior de las unidades domésticas remite a la posibilidad de tomar decisiones productivas directamente vinculadas a la producción agropecuaria, así como incidir en las decisiones generales de la familia. En el caso de las familias colonas, donde unidad de producción y reproducción se solapan, la relevancia de la titulación y el control de la tierra se incrementan ya que afecta la totalidad de las relaciones familiares. Contrario a lo que suponen algunos modelos económicos y legales, la bibliografía ha mostrado la ausencia de armonía al interior de los núcleos familiares. La existencia de diferentes intereses en términos de producción, reproducción e inversión a su interior supone que estas unidades constituyan espacios de negociación tanto hacia adentor como hacia el exterior (Agarwal, 1994). 
Agarwal (1994) sostiene que existen cuatro argumentos que colocan el tema de la propiedad de la tierra como una prioridad de género, a saber, los elementos vinculados al bienestar, a la eficiencia, a la igualdad y al empoderamiento. Deere y León (2001) toman y expanden dichos argumentos para el análisis del caso latinoamericano y sostienen que las desigualdades en el acceso se relacionan con discriminaciones en las tres modalidades por las cuales se accede a tierra (herencia, Estado o compra), y que estas discriminaciones están asentadas en conductas y patrones a nivel de la familia, la comunidad, el Estado y el mercado. Las autoras, como Agarwal (1994), consideran que es imprescindible retomar la senda del análisis de redistribución y en particular en la sociedad rural. Consideran que el accionar sobre la dimensión material constituye un paso previo e inevitable del proceso de construcción de igualdad en el reconocimiento.

A nivel latinoamericano los movimientos de mujeres han colocado la reivindicación por la tierra en la agenda pública y en particular a nivel internacional, empero son escasas las experiencias de políticas estatales que han recibido y jerarquizado estas demandas. En Uruguay, que se rige por un reglamento general de apropiación privada de la tierra, el acceso a la propiedad de la misma es sinónimo de acceso al sustrato de la unidad de producción y reproducción en el caso de las familias colonas. En consecuencia, la propiedad aparece como un factor que determina las posibilidades de producción económica, de sobrevivencia y determina en gran medida el nivel de bienestar y planificación futuro del núcleo familiar. El derecho de propiedad, en este caso de la tierra, no sólo es una cuestión de acceso y usufructo presente, sino que se torna trascendente a la hora de tomar decisiones futuras. La ausencia de activos ha sido reseñada en múltiples ocasiones como un factor determinante a la hora de impedir la disolución del núcleo familiar o del matrimonio por voluntad de la mujer, y ha sido señalado como un elemento que juega un rol fundamental en la continuidad de las situaciones de violencia doméstica. Asimismo, en las situaciones en las cuales el núcleo o matrimonio se disuelve por voluntad masculina se ha reseñado la profunda vulnerabilidad de la mujer al carecer de activos capaces de permitirle cierto nivel de condiciones de sobrevivencia. Deere y León (2001) sostienen que la forma como las mujeres pueden acceder a tierra son esencialmente tres, a saber, la compra, la herencia y la designación estatal vía política de tierras. A nivel internacional la literatura especializada señala que la principal vía de acceso a tierra de las mujeres es la herencia, mientras las políticas públicas y la compra directa inciden en menor medida.

Previo a que la reforma de colonización se procesara, y encuadrado en el marco legal y reglamentario antes descripto, Uruguay aparecía con fuertes sesgos de género en el acceso a la tierra, tanto en el general de la asignación y titulación, como en lo específico de las políticas colonizadoras. Para el año 2000, la distribución media en el Uruguay señalaba que el 18\% de los predios se encontraba en manos de mujeres (CGA, 20004) mientras que cuando se consideraba únicamente los predios del Instituto Nacional de Colonización -única política de tierras uruguaya- el porcentaje de predios en manos de mujeres descendía a 13,8\%. Cinco años después, aún sin los efectos de la reforma, el análisis del Censo de Colonos y Colonias realizado por el Instituto Nacional de

\footnotetext{
${ }^{4}$ Censo General Agropecuario, MGAP. Elaboración propia.
} 
Colonización para el año 2005 mostraba que la inequidad en el acceso a tierras públicas en el formato de apropiación privada de predios (fracciones de explotación individual) continuaba teniendo fuertes sesgos de género. Como lo ilustra el Gráfico 3, de las 3370 explotaciones registradas en el Censo existen 3074 que cuentan con una persona física como titular. De ellas 2535 $(82,6 \%)$ tienen titulares varones y $536(17,4 \%)$ cuentan con titulares mujeres.

\section{Gráfico 1. Censo de Colonos/as y colonias}

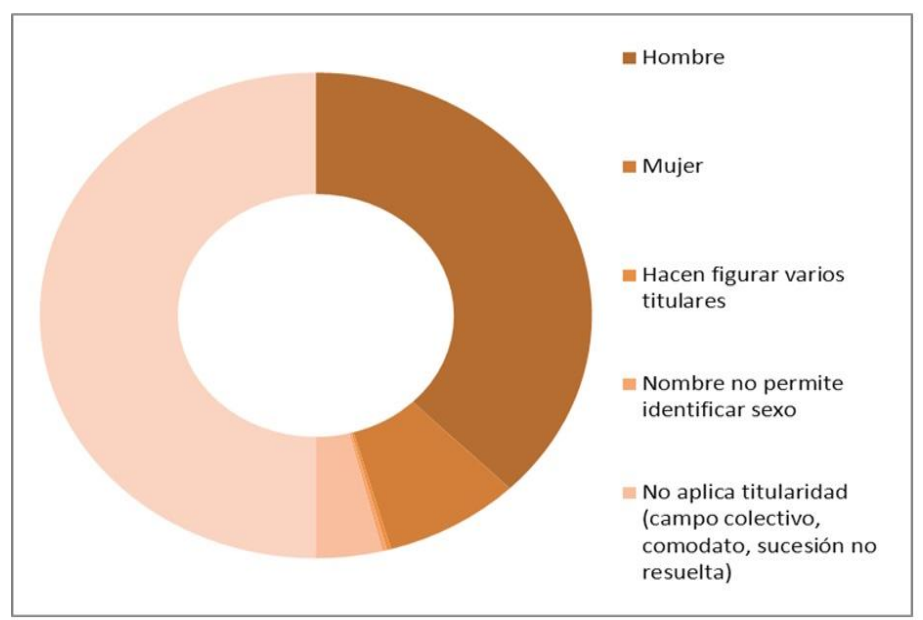

Fuente: Elaboración propia a partir del Censo de INC 2005 - $2006^{5}$

Debe considerarse que el Censo General Agropecuario y el Censo de Colonos y Colonias del INC arrojaron guarismos diferentes, y que ellos probablemente se vinculen con los 5 años que transcurrieron entre ambos -años en que se disparó el mercado de tierras en Uruguay- y con el hecho de que ambos relevamientos contaron con metodologías disímiles. Más allá de ello, es necesario visualizar que el sexismo en la propiedad de la tierra y en la política de Estado es evidente en ambos. Añádase asimismo, que cuando se considera el promedio de hectáreas, el CGA señala en el año 2000 que para INC la media de las mujeres es 143,61 há, mientras el promedio para los hombres es 199,13 há. Esta brecha se mantiene en el Censo de Colonización que indica una media de superficie en 2005 - 2006 de 152,8 hás para los varones y 127,7 hás para las mujeres.

\footnotetext{
${ }^{5}$ El Censo del Instituto Nacional de Colonización no registró el sexo de los/las colonos/as sino que identificó en cada fracción al/a la titular mediante su nombre, apellido y cédula. El procesamiento propio realizado supuso categorizar a partir de los nombres el sexo de las personas, sin embargo, existe un $0,6 \%$ de los registros en los que figuran nombres que pueden ser asignados tanto a varones como a mujeres. Asimismo, existe un 0,5\% de los registros en que se registraron como titulares a más de una persona, aún cuando por la reglamentación de INC sólo uno/a puede ser titular. Finalmente, hay un 7,7\% de los registros en los que la fracción no tiene una persona que esté asumiendo la titularidad ya sea porque es un comodato a un ente público u organización, porque se trata de una explotación colectiva del modelo anterior (ej. Campo de recría) o porque hay un proceso sucesorio en trámite.
} 


\section{Gráfico 2. Media de superficie de colonización, por sexo}

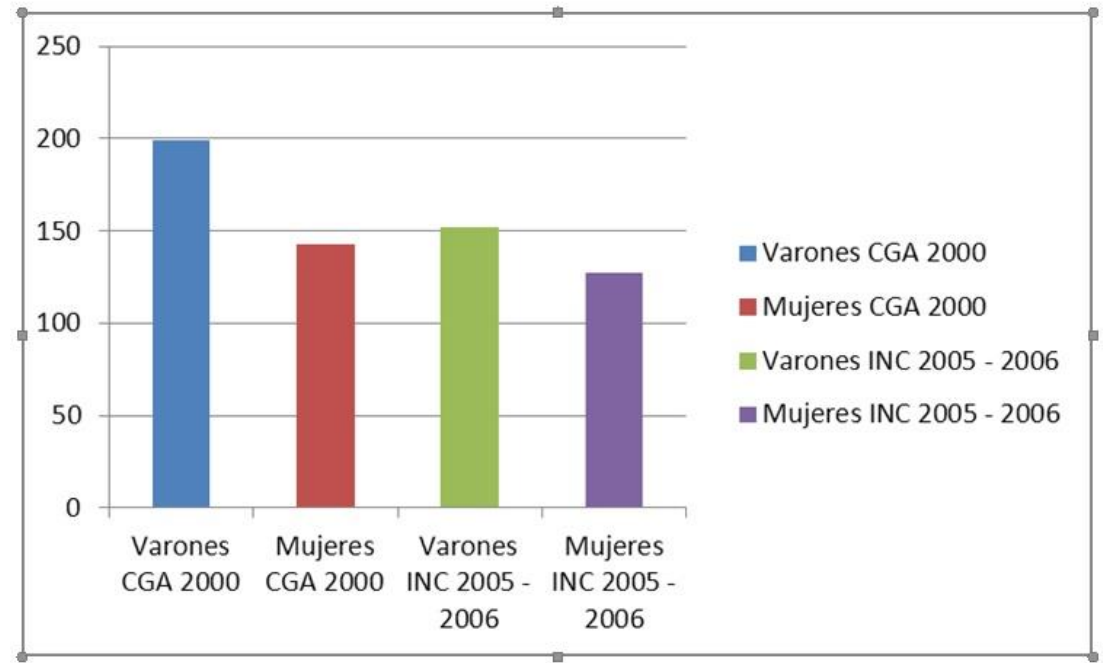

Fuente: Elaboración propia a partir de CGA - MGAP (2000) y Censo INC (2005 - 2006)

Estos sesgos en la tenencia de la tierra, adicionados a la forma como el INC concebía el proceso colonizador ponen de manifiesto que el periodo de la colonización previa al 2005 no contaba con una perspectiva de género, sino que lejos de ello introducía sesgos sistemáticos en el acceso por parte de mujeres y varones. De modo que, a 50 años de aplicación del modelo de la parcela individual para un colono y su familia, Uruguay arribaba al año 2005 con marcadas inequidades de género en la colonización.

\section{De la reforma ciega a la mirada de género}

En el año 2005 la llegada al gobierno del partido de izquierda, Frente Amplio, supuso una transformación en varios aspectos del quehacer institucional del Estado. En lo referido a esta temática, la fuerza política impulsó dos cambios considerados centrales para este artículo: la reforma del INC y el impulso a las transversalización de género en el Estado. En referencia a esto último, se señalará sucintamente la conformación del Consejo Nacional de Género como réctor en las políticas de género del país, y la construcción de un Plan de Igualdad de Oportunidades nacional, siendo el impulso en términos de género un elemento constatado en una amplia bibliografía institucional y académica (Pérez, 2007).

Por otra parte, a partir del 2005 se impulsó una reforma profunda del INC que se orientó a jerarquizar el instituto y a capitalizar al mismo, buscando con ello revertir una orientación del gobierno precedente que había encaminado la gestión del INC hacia su cierre y la 
liquidación de su cartera de tierra (Vassallo y Taddeo, 2009). Esta reforma se asentó en tres pilares, a saber:

(i) la gestación de una política activa de distribución a través del incremento en el presupuesto al INC para la expansión de la cartera de tierras del Instituto;

(ii) la reestructuración del propio Instituto desarrollando una nueva estructura organizacional e incorporando recursos humanos calificados, dando un mayor peso del funcionariado técnico - profesional en la composición del personal;

(iii) la realización de experiencias asociativas como estrategia colonizadora, de modo que una parcela fuera otorgada a un grupo para un usufructo colectivo en lugar de las precedentes parcelas individuales otorgadas a familias (Vassallo, Fernández y Rodríguez, 2010; Vassallo y Taddeo, 2009).

En este marco de reforma, el Instituto Nacional de Colonización tiene un avance en su presupuesto y compra de tierras, alcanzando el período 2005 - 2010 la compra de 47.053 hás ${ }^{6}$, compra comparable únicamente con el acto fundacional de la institución.

De modo que el marco de la reforma se vincula estrechamente con el contexto político nacional, específicamente con la llegada del partido Frente Amplio al gobierno nacional. La reforma concretada, entendida como producto de un contexto socio histórico particular (Guzmán y Montaño, 2012), debe ser concebida como el resultado final en un escenario de tensiones. Como se ha dicho, esta tranformación surge como una alternativa ante un proyecto colonizador que estaba siendo fuertemente cuestionado, y como estrategia de "salvataje" del INC que, en el período de gobierno precedente, había empezado un proceso de liquidación de su cartera de tierras como vía para la liquidación de la institución. El proceso resultante demostró que el principal eje de preocupación del gobierno, los/las directivos/as entrantes y la mayor parte del funcionariado era defender el proyecto colonizador, la sobrevivencia de la institución y de las fuentes de trabajo.

La reforma del INC hizo de las experiencias asociativas la política principal de adjudicación de tierras por parte del Estado, es decir, el acceso a tierras en forma colectiva pasó

\footnotetext{
6 "En lo que respecta a la incorporación de tierras en la nueva colonización, el Directorio anterior incorporó al INC, en cinco años de gestión, un total de 47.058 hás, incluyendo adquisiciones y traspasos de campos privados y estatales. Eso da un promedio de incorporación de tierras por año de 9.411 hás. Esto es, tomando únicamente la incorporación de tierras y descontado el traspaso inicial del BHU, más de una vez y media el promedio histórico de 57 años anterior a la pasada administración (promedio que ascendía a 5.914 hás por año). Si además del traspaso inicial del BHU se descuentan las ventas, en la administración precedente, la incorporación efectiva de tierras (incorporación menos ventas), por año, ascendió a 9.100 hás, contra un promedio anterior de 57 años de 2.077 hás /año, es decir, cuatro veces y media más que el promedio histórico" (INC, 2011: 6).
} 
a ser la vertiente principal de la institución ${ }^{7}$. Esta revisión supuso una expansión de la estructura funcionarial y la incorporación de nuevos equipos técnicos y profesionales, especialmente vinculados a las experiencias asociativas. Debe señalarse que aún en un contexto de impulso de la institucionalidad de género en el país, la reforma de la estructura no generó cargos ni ámbitos dentro de la institución destinados a la transversalización.

Congruentemente, como parte de la reglamentación y puesta en práctica de esta reforma, se estructura en 2010 un plan estratégico para el período 2010 - 2014 que jerarquiza el trabajo en las experiencias colectivas y reafirma la capacidad de estas experiencias de atender en mayor medida las necesidades y demandas de las poblaciones más vulnerables. Si bien en 2010 aparecen en el plan estratégico por primera vez dos menciones explícitas a las mujeres, estas menciones se realizan a través de ítems no jerárquicos del plan y no se traducen en actividades e indicadores de cumplimiento del mismo, por lo que en la práctica no tuvo en el plan 2010 - 2014 asignaciones presupuestales ni recursos humanos específicos.

De esta manera se proceso desde 2005 una reforma ciega al género, evidente en los ejes vertebradores de la reforma y en las metas que la institución se fija en términos de su competencia. De modo que a pesar de que los años 2005 a 2010, contexto del nuevo gobierno, aparecen como un tiempo de fuerte sensibilidad hacia la cuestión de género y su jerarquización institucional a través de Inamu/Inmujeres (Pérez, 2007; Beramendi, 2012), la reforma no hace de esta mirada un eje transversal de su reestructuración.

Lejos de eso, parece cumplirse en el proceso lo señalado por Franken (2007) quien señala que en la experiencia concreta el éxito de la transversalización de género ha estado subyugado a contar con un pre requisito de las políticas e institucionaes de contar con la seguridad de sobrevivencia, certeza que sólo se tuvo hasta el logro del segundo gobierno del Frente Amplio (2010).

Si el primer periodo de gobierno el INC aparece como un proceso ciego al género, el año 2013 se presenta como una verdadera revolución de género interna, constituyendo un punto de inflexión en la institución en términos de esta perspectiva. Asume por primera vez la presidencia del Instituto una mujer, y con esto se catalizan procesos claves en la incorporación de esta perspectiva en la institución como ser:

(i) la aprobación de la cotitularidad de la tierra para los predios que se entregan como parcelas individuales (no experiencias asociativas) a fines de 2014;

(ii) el reconocimiento de un trabajo en género y específicamente con mujeres rurales de funcionarios/as con formación específica;

(iii) la integración en 2015 del INC al Consejo Nacional de Género;

\footnotetext{
${ }^{7} \mathrm{El}$ acceso a tierras individuales se ha relegado a una posición marginal dentro del proceso colonizador, aplicándose esta estrategia a predios de pequeña escala que son devueltos al INC o pertenecientes a colonias de parcelas individuales pre existentes.
} 
(iv) la formación en género de un alto porcentaje de funcionarios/as de la institución;

(v) la aprobación en 2016 de una línea presupuestal vinculada al trabajo con colectivos de mujeres rurales (INC, 2016).

Este proceso coloca a la institución como referente en temas de género en el medio rural y es reconocida a nivel nacional e internacional (CEDAW, 2016) por el impacto que tiene la medida de la cotitularidad en el acceso de las mujeres a las parcelas de tierra individual.

\section{El nuevo modelo colonizador: las experiencias asociativas}

Como se señaló, hasta el 2005 el INC otorgaba tierras individualmente ya fuera en modo de propiedad o arrendamiento. Varios/as colonos/as y sus familias ocupaban determinada área de tierra sub-dividida en parcelas o chacras las cuales eran administradas individualmente. Si bien dichas colonias podían constituirse en comunidades agrarias ${ }^{8}$, el manejo y el uso de la tierra eran individuales. La nueva política promueve un estilo de colonias de tipo asociativo en las cuales los/as colonos/as comparten el uso y la administración de la tierra, participan de un predio colectivo.

Esta nueva política asociativa está destinada a grupos de productores y trabajadores rurales que no posean tierras o que requieran de mayor extensión para su desarrollo. Una de las principales modificaciones es que la distribución modifica su espíritu anterior de uso individual familiar, en estas nuevas experiencias no hay personas individuales que administran la tierra, sino que es un grupo y en algunos casos varios grupos quienes la gestionan.

Para la adjudicación colectiva, en muchas ocasiones el vínculo del INC es con los grupos y éstos/as priorizan los/as potenciales beneficiarios/as de entre su padrón de integrantes, para que luego de un análisis técnico el INC acepte el listado de personas adjudicatarias. Es decir que un grupo de la sociedad civil u organización es objeto de la política y es ésta la que pre selecciona quiénes de sus asociados/as podrán usufructuar la tierra de entre un listado de socios/as aspirantes, aprobando u observando posteriormente el INC el listado preparado por la organización.

"En el marco de las nuevas políticas colonizadoras impulsadas por el INC se promueven los emprendimientos asociativos y el surgimiento de empresas colectivas en diversos rubros (sobre todo en el ganadería, lechería y caña de azúcar) con la intención de incorporar tecnología aplicada a los procesos de producción (intensificar la producción ganadera, introducción de tecnologías para cosecha en rubros agrícolas, etc.). Por lo general, los emprendimientos se estructuran con dos niveles de asociatividad, en donde las colonias están divididas en fracciones, en las cuales el beneficiario es un

\footnotetext{
${ }^{8}$ Conglomerados de predios individuales que configuran una "colonia".
} 
colectivo (intra colectivo), al mismo tiempo, en cada colonia se establecen y disponen bienes e infraestructura productiva que es de uso común, promoviendo la articulación de los colectivos entre sí en diversas áreas (inter colectivo), estimulando la emergencia organizaciones de segundo grado. Los sujetos y protagonistas principales de esta nueva etapa son grupos de trabajo, cooperativas agrarias y de trabajo asociado, asociaciones y sociedades agrarias, sociedades de fomento rural, entre otras formas jurídicas existentes conformadas principalmente por trabajadores rurales y pequeños productores familiares" (Sitio Web INC/Información técnica y estadística/procesos colectivos).

Los grupos se constituyen entonces como "suma" de personas, "suma" de colonos/as. Como en el modelo precedente, se encuentra implícito en la política que junto a cada colono/ $\mathrm{a}^{9}$, advenirá la generación de bienestar y acceso del resto de la familia. Evidente esto en que en la nueva reglamentación se especifica que a efectos de las inscripciones en los grupos se debe registrar como "colono/beneficiario" únicamente una persona por familia, en consecuencia, la representación familiar a partir de un/a titular se mantiene como sucedía en la colonización anterior, previo al proceso de cotitularidad de las parcelas individuales.

"Número de integrantes: se estima viable un número de entre 5 y 20, de ser menor a 5 se deberán inscribir en forma individual aclarando en cada una de sus fichas que quieren trabajar en conjunto. En caso de grupo familiar, se deberá tener en cuenta que el postulante en sí y su familia ya constituyen el grupo, figurando solo el titular" (INC, 2012: 1).

Para la adjudicación se realiza un análisis técnico por parte de funcionarios/as de INC donde se valoran aspectos vinculados a la propuesta económico - productiva y su viabilidad, así como el hecho de que las personas postulantes sean del perfil priorizado por INC: pequeños/as productores y asalariados/as rurales.

"El formulario está integrado por varios cuadros cuyo fin es el relevamiento de datos de los grupos para el estudio de adjudicación y posterior seguimiento. El grupo deberá proporcionar los datos requeridos en el formulario de acuerdo a los siguientes ítems: A) Identificación del grupo y sus miembros, B) Orígenes e historia, C) Estructura, organización y dinámica de funcionamiento, D) Capacitación, E) Asesoramiento técnico recibido por el grupo, F) Trayectoria del grupo, G) Aspectos

\footnotetext{
${ }^{9}$ En cuanto al perfil de colonos la nueva reglamentación comprende al perfil establecido en 1948 aunque "estableciéndose que a los fines de la presente ley se deberá dar especial prioridad a las familias integradas por personas jóvenes y con niños en edad escolar, así como a los pequeños productores organizados, trabajando en grupo, que ya estén realizando explotaciones asociativas de la tierra, que exploten áreas insuficientes y/o con tenencias precarias." (Art. 13, Ley 18.187) El nuevo perfil de colono, como puede observarse, no destaca a las mujeres como objeto de adjudicación de tierras, sino que presupone su incorporación a través del genérico rol de colono -que ya se ha visto distribuye inequitativamente- o en su rol de madre o integrante de un núcleo familiar. Debe señalarse asimismo que en diferentes documentos generados por INC se intercala el concepto colono con el de beneficiario o productores beneficiarios, entendiéndose que en el caso de las experiencias individuales pervive la figura de colono únicamente y en el caso de las experiencias asociativas quien coloniza es un grupo o colectivo que, según los documentos, está compuesto por colonos, productores o beneficiarios. En todos los casos subsiste una figura individual que ingresa al grupo o experiencia asociativa como miembro o integrante en representación de su familia.
} 
económicos-productivos, H) Identificación de los miembros del grupo y de sus hogares, I) Sobre la edad, ocupación y grupo familiar, J) De la tenencia, uso y manejo de la tierra que poseen (cuadro para ganaderos y lecheros, para horticultores u otros rubros específicos crear cuadro complementario), K) Detalle de semovientes de propiedad del solicitante, L) Maquinaria y equipos referentes a la producción agropecuaria. La opinión de la Regional (o a quien se le asigne la tarea): se realizará tomando como referencia los criterios expuestos en el Procedimiento de selección de colonos en forma grupal (DEA, febrero de 2012), se opinará en consecuencia y según lo especificado en el formulario" (INC, 2012: 3).

Debe señalarse a su vez que una diferencia de la actual reglamentación con la precedente es el hecho de que se generan nuevas intermediaciones entre la ley de colonización y sus destinatarios/as. Concretamente, si en el pasado la Ley 11.029 (ley de colonización) podía establecer una figura de colono/a e imputar ciertos requisitos a su familia, la nueva estrategia colonizadora exige a los grupos de colonos/as que cuenten con una personería jurídica (cooperativas, sociedades de fomento, asociaciones agrarias) que son figuras jurídicas que ya se encuentran legisladas y que no prevén especificaciones sobre los derechos de las familias frente al/ a la titular de la figura jurídica.

Un último elemento significativo para caracterizar esta forma de colonización es que la misma no implica necesariamente que los/as colonos/as habiten el predio. De hecho, al ser predios colectivos la mayor proporción de los/as colonos/as no se radica en el predio ni tienen proyección de hacerlo. De esta forma se disloca la unidad productiva de la reproductiva, componente típico de la producción familiar y campesina.

\section{El nuevo formato colonizador frente al acceso y control de la tierra por parte de las mujeres}

Para analizar los impactos en términos de género del modelo de colonización asociativo es necesario superar la mirada unívoca a la titularidad y contemplar el acceso y control de la tierra. El enfoque de acceso y control sobre la tierra pone de manifiesto que la titularidad y sus indicadores no alcanzan para comprender a cabalidad el proceso de desigualdad que sufren las mujeres rurales.

Por el contrario, es necesario superar el modelo unidimensional de la tenencia para identificar procesos más complejos como las asimetrías que además se presentan en el uso y control. En consecuencia, se establece en primer lugar una distinción entre derecho y acceso. El derecho aparece como la formalización legal que habilita y legitima las decisiones en torno al uso de la tierra. El derecho está amparado en una forma de legitimación y de control, por ende, cuenta con una seguridad legal y una potencial capacidad de coacción. 
El acceso en cambio supone la posibilidad de uso en condiciones no necesariamente seguras ni estables, es posible acceder a la tierra en forma temporal, por ocupación o préstamo (Deere y León, 2002). El derecho efectivo a la tierra supone que a la previsión legal la acompaña un reconocimiento social que asegura que exista no sólo un derecho formal sino un control efectivo. El control efectivo, por su parte, supone el control de uso y sus beneficios, esto es, dominio sobre el proceso productivo y los resultados de la producción.

Partiendo desde este enfoque teórico fue necesario generar información inexistente en Uruguay, dado que los Censos Agropecuarios y el Censo de Colonos carecen de esta mirada. De modo que, a efectos de analizar la persistencia de desigualdades de género en el modelo de experiencias asociativas, fue necesario generar una encuesta donde se reflejara el acceso, control y uso de la tierra.

Para ello se realizó una encuesta a las experiencias asociativas de colonización de la Regional Salto, regional caracterizada por ser una de las de mayor cantidad de experiencias asociativas. La encuesta, realizada a efectos de esta investigación, se realizó durante el trimestre comprendido entre diciembre de 2013 y febrero de 2014, y relevó a 126 núcleos familiares vinculados a las colonias a través de un/a referente de la familia. La encuesta indagó en la situación de 322 personas adultas integrantes de 126 núcleos familiares, 169 varones y 153 mujeres.

La encuesta no sólo se centró en la concepción de titularidad, sino que adicionalmente indagó en torno a los conceptos de participación en los ámbitos de decisión (reuniones de grupo) y trabajo efectivo en la producción dentro del predio, que son identificados como centrales en la bibliografía especializada y que no se encuentran relevados en las fuentes existentes. Lamentablemente los indicadores relacionados a las decisiones y al trabajo productivo, no pueden ser comparados con guarismos de las experiencias anteriores de colonización ya que no existe información estadística al respecto.

El análisis de la muestra pone de manifiesto que esta nueva forma de colonización ha logrado reducir la brecha en el acceso a la tierra de colonización, pero que se mantiene importantes sesgos de género.

En el caso de las experiencias asociativas la condición de colono/a es producto de la titularidad de la figura jurídica adjudicataria y de la titularidad de la producción que se desarrolla dentro del predio, es decir, es beneficiario/a de la política quien figura como colono/a en la persona jurídica y produce dentro del predio. Si bien la política supone que estas condiciones se den simultáneamente, aparecen en la práctica algunas excepciones.

La Tabla 1 evidencia que entre las 322 personas integrantes de las 126 familias vinculadas a las experiencias asociativas existe una marcada tendencia a que los varones titulen la forma asociativa y posean producción propia en el campo colectivo, mientras en el caso de las mujeres el porcentaje es significativamente menor. 
Tabla 1. Acceso a la tierra según sexo en experiencias asociativas de la Regional Salto de INC

\begin{tabular}{|l|l|l|l|l|}
\hline & \multicolumn{2}{|l|}{ Titular de Producción } & \multicolumn{2}{l|}{ Titular de organización/grupo } \\
\hline & Hombre & Mujer & Hombre & Mujer \\
\hline Titular & $\mathbf{6 3 , 3 \%}$ & $\mathbf{2 3 , 5 \%}$ & $\mathbf{7 1 , 0 \%}$ & $\mathbf{3 2 \%}$ \\
\hline No titular & $36,7 \%$ & $76,5 \%$ & $29,0 \%$ & $68 \%$ \\
\hline Total & $100 \%$ & $100 \%$ & $100 \%$ & $100 \%$ \\
\hline
\end{tabular}

Fuente: Encuesta a participantes de experiencias asociativas de la Regional Salto de INC, 2013

En consecuencia, si bien las experiencias asociativas señalan una mejora en los porcentajes de la modalidad precedente, en esta modalidad persisten importantes sesgos, donde adicionalmente emergen diferencias entre el acceso a la figura de colono a efectos del INC y la verdadera posesión de la producción, razón de la adjudicación en un contexto donde el grupo sólo produce en la tierra, no la habita.

Asimismo, el relevamiento realizado en la Regional Salto de INC evidencia que no sólo la titularidad sesga el derecho de las mujeres a la producción agropecuaria, sino que aún en condiciones de titularidad la posibilidad de ser partícipes de las decisiones es desigual. A este respecto la Tabla 2 pone de manifiesto que las brechas en la titulación como derecho formal se acompañan de sesgos en el acceso a los ámbitos de decisión (reuniones del grupo) así como al trabajo efectivo en la producción del predio.

Tabla 2. Acceso a la tierra según sexo en experiencias asociativas de la Regional Salto de INC

\begin{tabular}{|l|l|l|l|l|}
\hline & \multicolumn{2}{|c|}{ Participación en reuniones } & \multicolumn{2}{c|}{ Trabajo en el predio } \\
\hline & Hombre & Mujer & Hombre & Mujer \\
\hline Titular & $\mathbf{7 2 , 8 \%}$ & $\mathbf{5 1 , 0 \%}$ & $\mathbf{7 0 , 4 \%}$ & $\mathbf{3 0 , 1 \%}$ \\
\hline No titular & $\mathbf{2 7 , 2 \%}$ & $\mathbf{4 9 \%}$ & $\mathbf{2 9 , 6 \%}$ & $\mathbf{6 9 , 9 \%}$ \\
\hline Total & $100 \%$ & $100 \%$ & $100 \%$ & $100 \%$ \\
\hline
\end{tabular}

Fuente: Encuesta a participantes de experiencias asociativas de la Regional Salto de INC, 2013

Analizando la situación de 322 personas se puede visualizar que las mujeres tienen menor titulación de la producción, menor titulación de la forma organizativa que da acceso a la tierra, menor participación en los ámbitos colectivos donde se toman las decisiones productivas y menor participación directa en la producción. En consecuencia, en esta forma que disloca la unidad productiva de la reproductiva las mujeres tienen un sistemático apartamiento de la titularidad, el uso y control de la tierra.

Los guarismos precedentes develan una desigualdad persistente cuya evolución no es pasible de ser analizada dada la ausencia de mediciones anteriores. Esta brecha actual se constituye como 
un desafío, aún cuando sí se constata una mejora en los indicadores de titulación frente a la forma de colonización precedente. En este sentido existe una pequeña reducción de la brecha de género en la titulación, consistente con el análisis cualitativo realizado por Camors (2015) quien desde el INC ha señalado que las experiencias asociativas constituyen una oportunidad para las mujeres de acceder a la tierra, en tanto las aspirantías en colectivo logran compensar la ausencia de recursos que afecta a las mujeres en el Uruguay.

A pesar de ello, la persistencia de brechas pone de manifiesto que en términos de diseño la política asociativa presenta aún algunas limitaciones para la igualdad de género. A saber:

(i) El acceso de las mujeres a los grupos de aspirantes cuenta con históricos sesgos de género surgidos de patrones culturales sexistas existentes en la sociedad rural, en las organizaciones rurales de la sociedad civil y en la asistencia técnica extensionista, elementos que fueron ya constatados para el país en relación a la conformación de grupos productivos para el acceso a políticas públicas (Florit et al., 2013).

(ii) Actualmente la política mantiene la titulación individual de un referente familiar, aspecto ya superado en las parcelas no asociativas. Adicionalmente con el requisito de la figura jurídica la relación entre INC y las familias no se ve sólo mediada por la figura del/de la titular, sino que en la nueva normativa se adicionan las restricciones de inscripción y derechos de cada una de estas figuras legales colectivas -cooperativas, asociaciones agrarias, etc-.

(iii)La efectiva asignación depende de la postulación de un formulario y una selección donde el peso del proyecto productivo y su viabilidad es central. Esta evaluación se realiza a nivel de INC y entre sus criterios contempla el perfil social de los/las aspirantes, los antecedentes del grupo y la disponibilidad de capital y/o activos para realizar una explotación rentable de la fracción que permita un uso productivo y la generación de ganancia para el pago de la renta al INC. La evaluación reserva una ponderación para el sexo, pero la misma es marginal frente a condiciones materiales previas como la disposición de animales o recursos para volver el campo redituable, aspectos que en Uruguay cuentan con fuertes sesgos de género (González et al., 2013

(iv) La disociación del espacio productivo del reproductivo, forma típica de la unidad de la producción familiar y campesina, conlleva un alejamiento de la familia del espacio de la producción. En consecuencia, los mecanismos habituales de incorporación de la familia completa a los procesos de producción se diluyen en este nuevo formato, limitando parcialmente el acceso de las mujeres a la producción. 


\section{Gender mainstreaming y tierras públicas}

Bajo el enfoque del gender mainstreaming este trabajo se orientó a discutir la incorporación de la perspectiva de género en la política del Instituto Nacional de Colonización, y especialmente en la incorporación transversal en la vertiente principal de la política pública de tierras: las experiencias asociativas. Los apartados precedentes permitieron mostrar que el Instituto Nacional de Colonización es una institución pública en cuyo seno se ha comenzado a dar sensibles avances en materia de género a partir de 2013, pero que cuenta con una reforma re fundacional que se gestó ciega al género, y con una política principal que aún presenta desafíos en materia de equidad.

Frente a ello, y a modo de reflexiones finales, la perspectiva del gender mainstreaming permite aportar dos elementos para encuadrar los desafíos para una plena transversalización, las lecciones del triángulo de terciopelo (Woodward, 2002) y las de la oportunidad (Guzmán y Montaño, 2012).

Como se ha señalado el triángulo de terciopelo (Woodward, 2002) identifica tres pilares necesarios para el logro de la transversalización, entendidas como necesarias alianzas estratégicas imprescindibles. En el contexto estudiado, el primer vértice del triángulo está dado por el funcionariado y el directorio del INC. En los primeros momentos del proceso re fundacional la institución se constituía por un funcionariado fuertemente masculinizado e influido por un orden de género fuertemente machista vinculado a lo rural y lo agropecuario. En este sentido, el proceso que se inicia en 2013 aparece como una revisión de una hegemonía sexista que existía en el periodo de inicio de la reforma.

Por su parte, cuando se analiza otro vértice del triángulo de terciopelo, la academia, se advierte que Uruguay no contó en el período 2005 - 2012 con ninguna publicación académica vinculada al acceso a la tierra de las mujeres. Es recién en el año 2014 que González et al. (2013) difunden un estudio focalizado en esta temática específicamente. A partir de este año la producción vinculada a las mujeres rurales ha sido más abundante a nivel nacional, constatándose que la cuestión de género vinculada a la ruralidad ha tenido un crecimiento sustancial en la bibliografía académica (Florit, 2016).

Finalmente, el último vértice del triángulo lo conforma según Woodward (2002) los movimientos feministas. En el caso de Uruguay y de la temática en cuestión, la sociedad civil está prepresentada en el medio rural por las organizaciones rurales, las cuales tienen por ley un lugar en el directorio de INC. No obstante, la participación de la sociedad civil en el directorio ha tenido en el periodo considerado a representantes de la organización IPL. Esta organización no ha priorizado hasta la actualidad el tema de acceso a tierra para las mujeres acorde a sus comunicaciones públicas, y hasta el año 2016 no habían desarrollado actividades específicas vinculadas a la cuestión de género en sí. Por su parte las organizaciones nacionales de mujeres 
rurales (AMRU, Red de Grupos de Mujeres Rurales) no cuentan con representantes propios/as en el directorio ni tienen un ámbito estipulado de diálogo con el representante de IPL en el directorio de INC.

En este sentido el triángulo permite visualizar con claridad desafíos y propuestas pertinentes para avanzar en la cuestión de género en las políticas de colonización y los de la institucionalidad agropecuaria nacional, a saber: (i) el fortalecimiento de las organizaciones de mujeres rurales y de sus espacios y posibilidades de representación; (ii) el sostenimiento del interés y compromiso de la academia con las mujeres rurales y la equidad de género en este medio; (iii) la sensibilización y compromiso del funcionariado y de la dirigencia política de las instituciones con la perspectiva de la transversalización.

Por su parte, Guzmán y Montaño (2012) acercan una lectura de la oportunidad como un elemento sustancial en el avance de la cuestión de género. En este sentido la lectura del proceso de INC como una trayectoria aparece como clave para comprender el camino que podrían seguir los desafíos aún pendientes. Respecto a la oportunidad, debe visualizarse que al momnneto de la reforma el INC se ubica en el centro de dos tensiones. Por una parte, la reforma es re fundacional y surge como "salvataje" frente a la voluntad existente en el gobierno precedente de liquidar la cartera de tierras y suprimir el proyecto colonizador. Por otra parte, una tensión divergente en ese contexto lo constituyó el compromiso del Frente Amplio con la transversalización de la perspectiva de género en el Estado (Pérez, 2007; Beramendi, 2012). Como se ha señalado la etapa fundacional de la reforma siguió un camino salvacionista que no buscó incorporar esta mirada de género transversal, en este sentido pareciera que la reforma no fue vista como una oportunidad, sino que, como señala Franken (2007) la transversalización no tiene chances en contextos de amenaza a la sobrevivencia.

En este sentido, lo que en el momento de la reforma re fundacional apareciera como una limitación, a partir del año 2013 aparece como oportunidad. El contex to vinculado al cambio en el directorio cataliza procesos que funcionarios/as aislados/as habían buscado impulsar sin éxito en la etapa precedente. De esta forma el contexto de una nueva presidencia, por primera vez mujer desde la creación del INC, así como la existencia de un nuevo cambio de gobierno (tercer periodo del Frente Amplio) a partir de 2015, con un mayor empuje de la cuestión de género en el país y un fuerte trabajpo interinstitucional de visualización de las mujeres rurales en las políticas públicas dan una nueva impronta y contexto para que el género colonice. 


\section{BIBLIOGRAFÍA}

- Agarwal, Bina (1994): "Gender and Command Over Property: A critical gap in economic analysis and policy in South Asia" En: World Development, vol. 22, n. 10, pp. 1455-1478.

- Beramendi, Carmen (2012): "Una vista al mainstriming de género desde el contexto". Ponencia presentada al Congreso Uruguayo de Ciencia Política. Mdeo, 14 al 16 de noviembre de 2012.

Diponible

en: http://www.aucip.org.uy/docs/cuarto_congreso/13131712\%20-\%20Beramendi,\%20Carmen.pdf [21/12/2016].

- Camors, Verónica (2015): "Políticas de acceso a la tierra y desarrollo rural del Instituto Nacional de Colonización en Uruguay. La situación de las productoras familiares y asalariadas rurales y las alternativas a las desigualdades existentes (2014 - 2015)". México: FLACSO. Sin publicar, Tesis de Maestría en Políticas públicas y género.

- Deere, Diana y León, Magdalena (2001): Género, propiedad y empoderamiento: tierra, Estado y mercado en América Latina. Bogotá: Tercer mundo editores.

- Florit, Paula (2013) (coord.): Estudios de financiamiento rural y asistencia técnica con perspectiva de género. Mdeo: REAF - AECID.

- Florit, Paula (2014): "Reforma del Instituto Nacional de Colonización ¿Qué hay de nuevo para el género?”. Mdeo: FCS - DS. Sin publicar, Tesis de especialización en género y políticas públicas.

- Florit, Paula y Piedracueva, Maximiliano (2016): "Mujeres y cuestión agraria: Capitalismo, Explotación y Patriarcado”. En: Revista electrónica Hemisferio Izquierdo, nº. 7. Disponible en: https://www.hemisferioizquierdo.uy/single-post/2016/11/07/Mujeres-y-cuesti\%C3\%B3nagraria-capitalismo-explotaci\%C3\%B3n-y-patriarcado [21/12/2016].

- Franken, Martha (2007): "Politics and Research. Two parts of a velvet triangle?". Ponenci/a en la Conferencia Internacional de Igualdad de oportunidades y derechos. Roma, Octubre de 2007.

- González, Diana et al. (2013): Acceso, tenencia, uso y control de tierras con perspectiva de género. El caso uruguayo. Mdeo: REAF - AECID.

- Guzmán, Virginia y Montaño, Sonia (2012): Políticas públicas e institucionalidad de género en América Latina (1985 - 2010). Serie Mujer y Desarrollo. Santiago de Chile: CEPAL.

- INC (2010): Plan Estratégico del Instituto Nacional de Colonización 2010 - 2014. Mdeo.: INC.

- INC (2005 - 2016) “Actas públicas de resoluciones del directorio". Montevideo: INC. Disponible en: http://www.colonizacion.com.uy/content/view/975/165/ [21/12/2016].

- Incháustegui, Teresa y Ugalde, Yamileth (2004): Materiales y herramientas conceptuales para la transversalidad de género. México D.F.: INMUJERES.

- Pateman, Carole (1996): Críticas feministas a la dicotomía público - privado. Barcelona: Paidós. 
- Pérez, Verónica (2007): Ir a más. Monitoreo de lo actuado por el Instituto Nacional de las Mujeres. (Periodo de 2005 - 2006). Montevideo: CNS Mujeres.

- Rigat - Pflaum, María (2009): "Las tensiones implícitas en la transversalización de la perspectiva de género. Una reflexión crítica sobre la implementación de políticas con perspectiva de género desde el Estado". Disponible http://www.inmujeres.gub.uy/innovaportal/file/21682/1/46_rigatpflaum2009_tensiones_gender mainstreaming.pdf [1/10/2016]

- Rigat - Pflaum, María (2008): “Gender mainstreaming: un enfoque para la igualdad de género." En: Nueva Sociedad, $\mathrm{n}^{\circ}$. 218, pp. 40-56. Disponible en: http://www.inmujeres.gub.uy/innovaportal/file/21671/1/35_maistreaming.pdf [1/10/2016].

- Vassallo, Miguel y Taddeo, José (2009): "MGAP e INC juntos para un desarrollo rural con enfoque territorial.” En Carlos Paolino (Coord.): Anuario OPYPA. Mdeo: OPYPA - MGAP, pp. $351-356$.

- Vassallo, Miguel; Fernández, Emilio y Rodríguez, Héctor (2010): Nuevas formas de organización de la producción y el acceso a la tierra, en una estrategia de Desarrollo Rural con enfoque territorial. Ponencia presentada al VII Congreso Latinoamericano de Sociología Rural. Noviembre de 2010, Porto de Galinhas.

- Walby, Sylvia (2011): The future of feminism. Malden: Polity Press. . (2004): "Mainstreaming de género: uniendo la teoría con la práctica". Ponencia a las Jornadas Mainstreaming de Género: conceptos y estrategias políticas y técnicas. Andalucía, 26 y 27 octubre de 2004. Disponible en: http://pmayobre.webs.uvigo.es/master/textos/luz_varela/ponencia_swalby..pdf [1/10/2016].

- Woodward, Alison (2002): Going for gender balance. Bruselas: Consejo de Europa. 